\title{
Grundlagen der Bioethik
}

Um die im vorangegangenen Kapitel beschriebene Situation bewältigen zu können, ist es hilfreich, sich einige Grundzüge der Medizin- und Bioethik vor Augen zu führen. Das Thema Medizin- und Bioethik wurde und wird in der Vergangenheit in der Literatur schon sehr umfänglich diskutiert. Leider fehlt noch die weiterführende Umsetzung in den pharmazeutischen Bereich. Deshalb möchte ich hier ein paar Gedanken skizzieren, die uns als Pharmazeuten auch die Möglichkeit geben sollen, ethisch oder moralisch schwierige Situationen zu bedenken und dann die möglichst richtige Entscheidung zu treffen.

Vorgestellt werden hier kurz die Ausführungen von Tom L. Beauchamp und James F. Childress, zwei amerikanischen Philosophen an der Georgetown University Washington, die sich sehr ausführlich mit diesem Thema beschäftigt haben und vier Prämissen für die Medizinethik aufgestellt haben, die sich auch auf die Pharmazie übertragen lassen. Man nennt diese Prämissen nach der Universität, an der Beauchamp und Childress arbeiten bzw. gearbeitet haben „Georgetown Mantra“. Stark verkürzt lassen sich diese wie folgt wiedergeben [14]:

- Selbstbestimmungsrecht des Patienten (respect for autonomy)

- Prinzip der Schadensvermeidung (non-maleficence)

- Patientenwohl (beneficence)

- Soziale Gerechtigkeit (justice)

Diese schlaglichtartigen Ideen können kaum alle Gedanken reflektieren. Dennoch stellen sie eine Möglichkeit dar, in der täglichen Arbeit einen Rahmen zu geben, an dem man sich orientieren kann und der zum Nachdenken anregen soll. Die Inhalte der einzelnen Punkte wurden an verschiedenen Stellen dieses Buches schon diskutiert. 